\title{
Improvement of Dynamic Modelling for Single-phase Heated Tubes
}

\author{
Du Zhehua $^{1}$ \\ ${ }^{1}$ Wuhan Second Ship Design and Research Institute, 430205 Wuhan, China
}

\begin{abstract}
To improve the currently available multi-segment lumped parameter models, a new dynamic mathematic model for single-phase heated tubes is established by employing dynamic equations instead of the traditional static equations for calculating the heat transfer across the control volume's tube wall. Through separating the temporal and spatial terms of the partial differential equations, and combining both lumped and analytical methods, an analytical solution for the temperature distribution of the steam and water in the tubes is derived. Then, by integrating the elemental volume equations, dynamic equations for the heat release of the tube wall are achieved. The improved model, which can be explicitly solved, is more stable and has much wider applicability. After applying the improved model to an ultra-supercritical boiler superheater, the results show that the response characteristics for inlet temperature step change are significantly improved, and the results during the puredelay periods are in accordance with those of the distributed parameter models. Compared with traditional multi-segment models, the improved model can achieve higher precision even with less segments. Besides, the transport delay characteristics of water or steam within tubes can be analyzedmore accurately with the improved model
\end{abstract}

\section{Introduction}

Single-phase heated tube parameter modeling is one of the important research contents in the field of real-time simulation of power station boilers[1]. In dynamic process, due to heat storage and storage change between pipe wall and working medium, the heat released by pipe wall to working medium is generally not consistent with steady-state calculated value. Heat release between tube wall and working medium is basically calculated by static equation. This is not conducive to further improvement of heated tube simulation accuracy.

In order to improve the simulation accuracy of parametric model on a single control body, a modeling method based on dynamic compensation or correction has emerged since the 1990s[2]. By comparing and analyzing the difference in the system transfer function, a mathematical model is established to compensate or correct the difference[3]. In the selection of lumped parameters, "moving parameter" is adopted to correct the model transport delay.

In this paper, based on one-dimensional partial differential equation describing the dynamic process of single-phase heat pipe, a new dynamic mathematical model of single-phase heat pipe (hereinafter referred to as mixed model) is established by combining analytic and lumped parameters with space-time separation in the equation. Theoretical analysis and simulation results show that, under the same circumstances, mixed model is closer to distributed parameter model than previous lumped parameter model using static heat transfer formula.

\section{Modeling Principle}

\subsection{One-dimensional differential equations describing the dynamic process of single-phase tubes}

One-dimensional lumped parameter physical model is obtained by simplifying three-dimensional distributed parameter physical model in space. The physical model is shown in Figure 1. One-dimensional differential equation describing the dynamic process of a singlephase tube consists of the following equations[4].

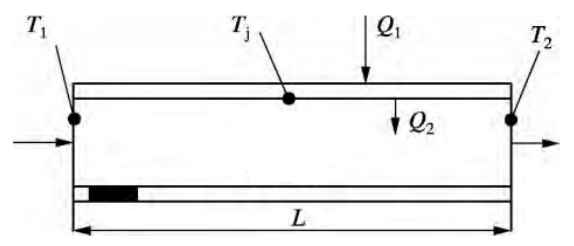

Fig. 1. Physical model of single-phase heating pipe

- Mass conservation equation (continuity equation)

$$
\frac{\partial D}{\partial z}+F \frac{\partial \rho}{\partial \tau}=0
$$

\footnotetext{
* Corresponding author: shunli878@163.com
} 
In the formula, $D$ is working fluid flow. $\rho$ is working fluid density. $z$ is the length. $\tau$ is the time. $F$ is tube's cross-sectional area.

- Energy conservation equation

$$
Q_{2}=D \frac{\partial h}{\partial z}+F \rho \frac{\partial h}{\partial \tau}-F \frac{\partial P}{\partial \tau}
$$

In the formula, $Q_{2}$ is the heat release of pipe wall to working fluid per unit length. $h$ is the working fluid enthalpy. $P$ is working pressure.

- The heat release equation of pipe wall per unit length to working fluid[5].

$$
\begin{gathered}
Q_{2}=\alpha_{2} \pi d_{2}\left(T_{j}-T\right)=\alpha_{2} U_{2}\left(T_{j}-T\right) \\
\approx K_{2} D^{n} U_{2}\left(T_{j}-T\right)
\end{gathered}
$$

In the formula, $T$ is the working fluid temperature. $T_{j}$ is the metal temperature of tube wall. $\alpha_{2}$ is the heat release coefficient of tube inner wall to working fluid. $n$ is $0.8 . K_{2}$ is proportional coefficient. $d_{2}$ is tube inner diameter. $U_{2}$ is the circumference of the tube inner wall.

- Momentum conservation equation

$$
\frac{\partial P}{\partial z}+P_{d}=0
$$

In the formula, $P_{d}$ is the pressure loss per unit length.

- Thermodynamic state equation

$$
\begin{aligned}
& \rho=\rho(P, T) \\
& h=h(P, T)
\end{aligned}
$$

- Tube wall metal heat balance equation

$$
Q_{1}-Q_{2}=m_{j} c_{j} \frac{\partial T_{j}}{\partial \tau}
$$

In the formula, $Q_{1}$ is heat release of flue gas to the tube wall per unit length. $c_{j}$ is specific heat. $m_{j}$ is metal mass per unit length.

2.2 Dynamic equation of heat release from tube wall and outlet temperature of work fluid.

The following chain expression can be obtained from Equation (6).

$$
\begin{aligned}
\frac{\partial h}{\partial z} & =\left(\frac{\partial h}{\partial T}\right)_{\mathrm{P}} \frac{\partial T}{\partial z}+\left(\frac{\partial h}{\partial P}\right)_{\mathrm{T}} \frac{\partial P}{\partial z} \\
& =C_{p} \frac{\partial T}{\partial z}-\left(\frac{\partial h}{\partial P}\right)_{\mathrm{T}} P_{d}
\end{aligned}
$$

In the formula, $c_{p}$ is the specific heat of working fluid. There are also the following expressions for $\frac{\partial h}{\partial \tau}$.

$$
\frac{\partial h}{\partial \tau}=c_{p} \frac{\partial T}{\partial \tau}+\left(\frac{\partial h}{\partial P}\right)_{\mathrm{T}} \frac{\partial P}{\partial \tau}
$$

Substituting equations (8) and (9) into equation (2), the following equation is obtained.

$$
\begin{array}{r}
\alpha_{2} U_{2}\left(T_{j}-T\right)=D c_{p} \frac{\partial T}{\partial z}-D P_{d}\left(\frac{\partial h}{\partial P}\right)_{\mathrm{T}} \\
+F \rho c_{p} \frac{\partial T}{\partial \tau}+F\left(\rho\left(\frac{\partial h}{\partial P}\right)_{\mathrm{T}}-1\right) \frac{\partial P}{\partial \tau}
\end{array}
$$

Using $\frac{\partial T_{j}}{\partial z}=0$, and the flow calculation formula $D=F \rho \omega$, the following formula is obtained.

$$
\begin{aligned}
& \frac{\partial\left(T_{j}-T\right)}{\partial z}+\frac{\alpha_{2} U_{2}}{D c_{p}}\left(T_{j}-T\right)=\frac{1}{\omega} \frac{\partial T}{\partial \tau}- \\
& \frac{P_{d}}{c_{p}}\left(\frac{\partial h}{\partial P}\right)_{\mathrm{T}}+\frac{F}{D c_{p}}\left(\rho\left(\frac{\partial h}{\partial P}\right)_{\mathrm{T}}-1\right) \frac{\partial P}{\partial \tau}
\end{aligned}
$$

In the formula, $\omega$ is the working fluid flow rate.

Considering that the dynamic process of pressureflow channel is much faster than that of enthalpytemperature channel, it is generally considered that the dynamic process of pressure-flow channel has been concluded when enthalpy-temperature channel is calculated. On the other hand, for the pressure-flow channel model where the resistance is concentrated at the inlet or outlet, the pressure in the tube is considered to be uniformly distributed along the space. Therefore, equation (10) is further simplified to obtain the following equation.

$$
\frac{\partial\left(T_{j}-T\right)}{\partial z}+\frac{\alpha_{2} U_{2}}{D c_{p}}\left(T_{j}-T\right)=\frac{1}{\omega} \frac{\partial T}{\partial \tau}
$$

Equation (11) is a time-varying nonlinear partial differential equation. If the equation right term at any $\tau$ time can be determined, the above equation can be transformed into a non-homogeneous differential equation with constant coefficients. Integrate and substitute into the boundary condition $\left(T_{j}-T\right)_{z=0}=T_{j}-T_{1}$, the solution is as follows.

$$
T_{2}=T_{j}-\frac{1}{\omega} \frac{D c_{p}}{\alpha_{2} A_{2}} \frac{d T^{*}}{d \tau}-\left(T_{j}-T_{1}-\frac{L}{\omega} \frac{D c_{p}}{\alpha_{2} A_{2}} \frac{d T^{*}}{d \tau}\right) e^{-\frac{\alpha_{2} A_{2}}{D c_{p}}}
$$

In the formula, $T_{1}$ and $T_{2}$ are the inlet and outlet temperature of working fluid in the tube. $L$ is the total length of heated tube. $\mathrm{A}_{2}$ is the tube inner surface area, $A_{2}=U_{2} L$.

Similarly, working fluid temperature at any position along the tube length can also be obtained by using equation (11). Substituting it into equation (3) and integrating it, the dynamic equation of heat release $\bar{Q}_{2}$ of tube wall to working fluid is obtained.

$$
\bar{Q}_{2}=D c_{p} \frac{L}{\omega} \frac{d T^{*}}{d \tau}+D c_{p}\left(T_{j}-T_{1}-\frac{L}{\omega} \frac{D c_{p}}{\alpha_{2} A_{2}} \frac{d T^{*}}{d \tau}\right)\left(1-e^{-\frac{\alpha_{2} A_{2}}{D c_{p}}}\right)
$$

Equations (7), (12) and (13) constitute a new dynamic mathematical model for single-phase heated tubes. In order to investigate the final steady-state value of mixed model, let time-varying terms $\frac{d T^{*}}{d \tau}$ in equations (12) and (13), the following two formulas are obtained.

$$
\begin{gathered}
T_{20}=T_{j o}-\left(T_{j 0}-T_{10}\right) e^{-\frac{\alpha_{20} A_{2}}{D_{0} c_{p 0}}} \\
\bar{Q}_{20}=D_{0} c_{p 0}\left(T_{j}-T_{1}\right)_{0}\left(1-e^{-\frac{\alpha_{20} A_{2}}{D_{0} c_{p 0}}}\right)
\end{gathered}
$$


In the formula, the subscript 0 represents the parameter in steady state. According to heat transfer theory, equations (14) and (15) are equivalent to the steady-state equation of heat transfer calculated by logarithmic heat transfer temperature difference, and have nothing to do with the choice of parameter $T^{*}$.

For example, working fluid outlet temperature is $T_{2}$, at this time the equations to be calculated are $d T_{2} / d \tau$, $d T_{j} / d \tau$ and $\bar{Q}_{2}$, which can be described as $A \cdot y=S$ in matrix form.

$$
\begin{aligned}
& A=\left(\begin{array}{ccc}
\frac{L}{\omega} \frac{D c_{p}}{\alpha_{2} A_{2}}\left(1-e^{-\frac{\alpha_{2} A_{2}}{D c_{p}}}\right) & 0 & 0 \\
D c_{p} \frac{L}{\omega}\left(1-\frac{D c_{p}}{\alpha_{2} A_{2}}\right)\left(1-e^{-\frac{\alpha_{2} A_{2}}{D c_{p}}}\right) & 0 & -1 \\
0 & M_{j} c_{j} & 1
\end{array}\right) \\
& y=\left(\begin{array}{c}
\frac{d T_{2}}{d \tau} \\
\frac{\partial T_{j}}{\partial \tau} \\
\bar{Q}_{2}
\end{array}\right) \quad S=\left(\begin{array}{c}
\left(1-e^{-\frac{\alpha_{2} A_{2}}{D c_{p}}}\right)+e^{-\frac{\alpha_{2} A_{2}}{D c_{p}}} T_{1}+T_{2} \\
-D c_{p}\left(1-e^{-\frac{\alpha_{2} A_{2}}{D c_{p}}}\right)\left(T_{j}-T_{1}\right) \\
\bar{Q}_{1}
\end{array}\right)
\end{aligned}
$$

Because of the obvious difference between hybrid model and traditional lumped parameter model in form, it is necessary to analyze its accuracy. In the following analysis and calculation, mixed model adopts the form of $T_{2}$ instead of $T^{*}$.

\section{Model accuracy analysis}

From basic equations (7), (12) and (13), the object transfer function of working fluid under the three disturbances of inlet temperature, heat flow and flow rate are obtained. Taylor expansion is performed at the zero point $(s=0)$ to examine the degree of approximation between mixed model and distributed parameter model, and the advantages of mixed model over traditional lumped parameter model.

\subsection{Transfer Function}

Respectively linearize equations (7), (12) and (13), and use time $\tau$ as independent variable to perform Laplace transformation to obtain the transfer function $W_{\eta}(s)$ when inlet temperature is disturbed.

$$
W_{\eta}(s)=\frac{\frac{a_{d} T_{m} e^{-a_{d}}}{1-e^{-a_{d}}} s+1}{\tau_{0} T_{m} s^{2}+\left(\tau_{0}+\frac{a_{d} T_{m}}{1-e^{-a_{d}}}\right) s+1}
$$

In the formula, $T_{m}$ is heat storage time constant of tube wall. $a_{d}$ is dynamic parameter, $a_{d}=\alpha_{20} A_{2} /\left(D_{0} c_{p}\right) . \tau_{0}$ is flow time, $\tau_{0}=L / \omega_{0}$.
The transfer function $W_{q}(s)$ when heat flow is disturbed is shown in the following equation.

$$
W_{q}(s)=\frac{1}{\tau_{0} T_{m} s^{2}+\left(\tau_{0}+\frac{a_{d} T_{m}}{1-e^{-a_{d}}}\right) s+1}
$$

The transfer function $W_{d}(s)$ in the case of flow disturbance is shown in the following equation.

$$
W_{d}(s)=-\frac{\frac{(1-n) a_{d}^{2} T_{m} e^{-a_{d}}}{\left(1-e^{-a_{d}}\right)^{2}} s+1}{\tau_{0} T_{m} s^{2}+\left(\tau_{0}+\frac{a_{d} T_{m}}{1-e^{-a_{d}}}\right) s+1}
$$

\subsection{Precision analysis}

In theory, time domain analysis is commonly used to compare and analyze the dynamic characteristics of the thermal process. By comparing Taylor expansion of transfer function at $s=0$, the degree of approximation between models can be qualitatively analyzed. For the response characteristics of the model at the initial moment of disturbance and the final value of steady state, the initial value theorem and final value theorem of Laplace transform can be used for analysis. The transfer function of the simplified distributed parameter model has the following form [6].

$$
\begin{aligned}
& \bar{W}_{\eta}(s)=\exp \left(-\tau_{0} s-\frac{a_{d} T_{m} s}{1+T_{m} s}\right) \\
& \bar{W}_{q}(s)=\frac{1-\bar{W}_{\eta}}{\tau_{0} T_{m} s^{2}+\left(\tau_{0}+a_{d} T_{m}\right) s} \\
& \bar{W}_{d}(s)=\frac{\left(1+(1-n) T_{m} s\right)\left(1-\bar{W}_{\eta}\right)}{\tau_{0} T_{m} s^{2}+\left(\tau_{0}+a_{d} T_{m}\right) s}
\end{aligned}
$$

In the formula, $\bar{W}_{\eta}(s), \bar{W}_{q}(s)$ and $\bar{W}_{d}(s)$ are the transfer functions of inlet temperature, heat flow and flow disturbance of distributed parameter model, respectively.

Under the same conditions, the transfer function of lumped parameter model is as follows.

$$
\begin{aligned}
& \overparen{W}_{\eta}(s)=\frac{T_{m} s+1}{\tau_{0} T_{m} s^{2}+\left(T_{m}+\tau_{0}+a_{d} T_{m}\right) s+1} \\
& \overparen{W}_{q}(s)=\frac{1}{\tau_{0} T_{m} s^{2}+\left(T_{m}+\tau_{0}+a_{d} T_{m}\right) s+1} \\
& \overparen{W}_{d}(s)=-\frac{(1-n) T_{m} s+1}{\tau_{0} T_{m} s^{2}+\left(T_{m}+\tau_{0}+a_{d} T_{m}\right) s+1}
\end{aligned}
$$

In the formula, $\overparen{W}_{\eta}(s), \overparen{W}_{q}(s)$ and $\overparen{W}_{d}(s)$ are the transfer functions of lumped parameter model inlet temperature, heat flow and flow disturbance, respectively.

According to Laplace's initial value theorem and final value theorem, it is easy to obtain that three transfer functions of mixed model are consistent with the results 
of corresponding transfer functions of distributed parameter model when $s \rightarrow \infty$ and $s \rightarrow 0$. This shows that mixed model has no errors in the initial and final values of dynamic response. Equations (16) (24) are expanded at $\mathrm{s}=0$. After omitting high-order terms, Taylor expansions of the three model inlet temperature disturbance transfer functions are obtained.

$$
\begin{aligned}
& \bar{W}_{\eta}(s)=1-\left(\tau_{0}+a_{d} T_{m}\right) s+\frac{\left(\tau_{0}+a_{d} T_{m}\right)^{2}+2 a_{d} T_{m}^{2}}{2 !} s^{2}+O\left(s^{2}\right) \\
& W_{\eta}(s)=1-\left(\tau_{0}+a_{d} T_{m}\right) s+\frac{\left(\tau_{0}+a_{d} T_{m}\right)^{2}+2 a_{d} T_{m}^{2}+\tau_{0}-a_{d}^{2} T_{m}^{2}}{2 !} s^{2}+O\left(s^{2}\right) \\
& \widehat{W}_{\eta}(s)=1-\left(\tau_{0}+a_{d} T_{m}\right) s+\frac{\left(\tau_{0}+a_{d} T_{m}\right)^{2}+2 a_{d} T_{m}^{2}+\left(\tau_{0}+a_{d} T_{m}\right)^{2}}{2 !} s^{2}+O\left(s^{2}\right)
\end{aligned}
$$

Taylor expansion of heat flow perturbation transfer function of three models is as follows.

$$
\begin{aligned}
& \bar{W}_{q}(s)=1-\left(\frac{\tau_{0}+a_{d} T_{m}}{2}+T_{m}\right) s+O(s) \\
& W_{q}(s)=1-\left(\tau_{0}+\frac{a_{d} T_{m}}{1-e^{-a_{d}}}\right) s+O(s) \\
& \approx 1-\left(\frac{\tau_{0}+a_{d} T_{m}}{2}+T_{m}+\frac{\tau_{0}-a_{d} T_{m}}{2}\right) s+O(s) \\
& \overparen{W}_{q}(s)=1-\left(\tau_{0}+a_{d} T_{m}+T_{m}\right) s+O(s) \\
& \quad=1-\left(\frac{\tau_{0}+a_{d} T_{m}}{2}+T_{m}+\frac{\tau_{0}+a_{d} T_{m}}{2}\right) s+O(s)
\end{aligned}
$$

Taylor expansions of three model flow disturbance transfer functions are as follows.

$$
\begin{aligned}
& \bar{W}_{d}(s)=-1+\left(\frac{\tau_{0}+a_{d} T_{m}}{2}+n T_{m}\right) s+O(s) \\
& \left.W_{d}(s)=-1+\left(-\frac{(1-n) a_{d}^{2} T_{m} e^{-a_{d}}}{\left(1-e^{-a_{d}}\right.}\right)^{2}+\tau_{0}+\frac{a_{d} T_{m}}{1-e^{-a_{d}}}\right) s+O(s) \\
& \approx-1+\left(\frac{\tau_{0}+a_{d} T_{m}}{2}+n T_{m}+\frac{\tau_{0}-(2 n-1) a_{d} T_{m}}{2}\right) s+O(s) \\
& \overparen{W}_{d}(s)=-1+\left(\frac{\tau_{0}+a_{d} T_{m}}{2}+n T_{m}+\frac{-\tau_{0}+a_{d} T_{m}}{2}\right) s+O(s)
\end{aligned}
$$

For the transfer function of inlet temperature disturbance, the comparison result on the second-order accuracy is as follows.

$\left|W_{\eta}(s)-\bar{W}_{\eta}(s)\right|=\left|\frac{\tau_{0}-a_{d}^{2} T_{m}^{2}}{2 !}\right|<\left|\frac{\left(\tau_{0}+a_{d} T_{m}\right)^{2}}{2 !}\right|=\left|\widehat{W}_{\eta}(s)-\bar{W}_{\eta}(s)\right|$

For the transfer function of heat flow perturbation, the first-order precision comparison result is as follows.

$$
\left|W_{q}(s)-\bar{W}_{q}(s)\right|=\left|\frac{a_{d} T_{m}-\tau_{0}}{2}\right|<\left|\frac{a_{d} T_{m}+\tau_{0}}{2}\right|=\left|\mathscr{W}_{q}(s)-\bar{W}_{q}(s)\right|
$$

For the transfer function of flow disturbance $(n=0.8)$, the first-order accuracy comparison result is as follows.

$$
\left|W_{d}(s)-\bar{W}_{d}(s)\right|=\left|\frac{0.6 a_{d} T_{m}-\tau_{0}}{2}\right|<\left|\frac{a_{d} T_{m}-\tau_{0}}{2}\right|=\left|\mathscr{W}_{d}(s)-\bar{W}_{d}(s)\right|
$$

The above analysis shows that mixed model is closer to the distributed parameter model than the lumped parameter model when the export parameter of working fluid is also selected as lumped parameter.

\section{Conclusion}

The mixed model is derived from one-dimensional partial differential equation describing the heat transfer and flow of heated tube. It is derived in the way of heat transfer differential element integration. This is consistent with the dynamic process mechanism of single-phase heated pipe.

The dynamic equation of heat release from tube wall reflects the influence of heat storage and mass storage process of working fluid in the tube on its calculated value. And its steady-state value is equivalent to the equation for calculating heat transfer based on the logarithmic heat transfer temperature difference. This makes the simulation accuracy of the model on a single control body better than that of the commonly used multi-segment lumped parameter model. Accuracy analysis shows that the simulation accuracy of mixed model is higher than that of traditional lumped parameter model even without considering changes in physical properties.

The simulation results show that, compared with the step response of inlet flow and heat flow, mixed model's improvement of step response of inlet temperature is particularly prominent. This can better reflect transport delay characteristics of system response

The selection of the working fluid lumped parameters in the tubes is independent. This brings a higher degree of freedom and versatility to the specific application of the model.

\section{References}

1. S. Chandrasekharan, R.C. Panda, B.N. Swaminathan, Reviews in Chemical Engineering, 30, 1 (2014)

2. S.B. Yan, J.J. Li, X.D. Zhang, Journal of Zhengzhou University, 33, 55 (2012)

3. G. Chen, T.J. Ren, Journal of Tsinghua University: Science \& Technology, 47, 695 (2007)

4. Q.J. Liang, J.Z. Liu, Y.G. Xue, Journal of Chinese Society of Power Engineering, 32, 118 (2012)

5. Z.N. Wang, Q.T. Zhou, Journal of Southeast University: Natural Science Edition, 24, 83 (1994)

6. E. Oko, M.H. Wang, Fuel, 135, 292 (2014) 